In Situ

Revue des patrimoines
In Situ

Revue des patrimoines

22 | 2013

La peinture murale : héritage et renouveau

\title{
Marcel-Lenoir et la fresque
}

\section{Marie-Ange Namy}

\section{OpenEdition}

Journals

Édition électronique

URL : http://journals.openedition.org/insitu/10755

DOI : 10.4000/insitu. 10755

ISSN : 1630-7305

\section{Éditeur}

Ministère de la culture

\section{Référence électronique}

Marie-Ange Namy, « Marcel-Lenoir et la fresque », In Situ [En ligne], 22 | 2013, mis en ligne le 12 novembre 2013, consulté le 20 avril 2019. URL : http://journals.openedition.org/insitu/10755 ; DOI : 10.4000/insitu. 10755

Ce document a été généré automatiquement le 20 avril 2019

\section{(2) $\oplus \Theta \Theta$}

In Situ Revues des patrimoines est mis à disposition selon les termes de la licence Creative Commons Attribution - Pas d'Utilisation Commerciale - Pas de Modification 4.0 International. 


\title{
Marcel-Lenoir et la fresque
}

\author{
Marie-Ange Namy
}

\section{Marcel-Lenoir : un initiateur dans la renaissance de l'art religieux et la résurrection de la fresque ${ }^{1}$}

L'artiste Jules Oury dit Marcel-Lenoir (Montauban, 1872-Montricoux, 1931) bijoutier, enlumineur, graveur, dessinateur, peintre et fresquiste est une figure oubliée qui compte pourtant dans l'histoire de l'art de la fin du XIX ${ }^{\mathrm{e}}$ et du début du XX $\mathrm{X}^{\mathrm{e}}$ siècle (fig. $\left.\mathbf{n}^{\circ} \mathbf{1}\right)$. Cette personnalité atypique noue des liens étroits avec l'ensemble des réseaux du monde littéraire et artistique. Sans s'enfermer dans une chapelle stylistique, il vit et évolue au cœur de toutes les avant-gardes. Tout au long de son cheminement artistique, il garde, en dépit de sa surprenante variété, une véritable homogénéité de parcours, guidé par une pensée idéaliste. L'adjectif «lumineux» pourrait caractériser l'art de cette figure hautaine et austère. 


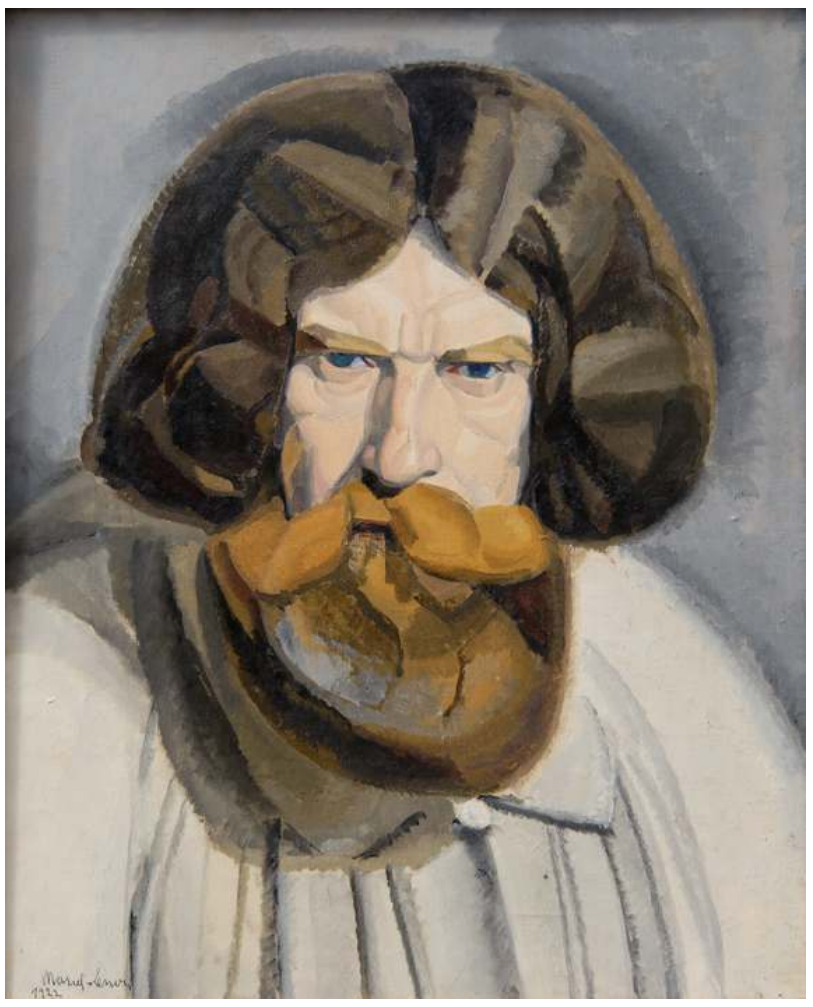

Marcel-Lenoir : Autoportrait (Marcel-Lenoir en blouse de fresquiste), huile sur toile, 1922, collection particulière.

Phot. Namy, Jean-Baptiste, 2011. ( ) Archives Musée Marcel-Lenoir.

2 L'artiste évolue au cœur de ce formidable et grandiose renouveau de l'art sacré, qui renaît à la fin du XIX ${ }^{\mathrm{e}}$ siècle pour s'épanouir tout au long du XX $\mathrm{XX}^{\mathrm{e} 2}$. Dans la résurrection de l'art de la fresque, il se révèle alors comme un acteur passionné et un fresquiste singulier parmi quelques artistes qui s'engagent et fondent, pour certains, leur propre école. L'histoire de la fresque au XX siècle grave alors le nom de Paul Baudoüin (1844-1931). À partir de 1911, Paul Baudoüin a donné un cours de fresque à l'école des Beaux-Arts. Puis une chaire a été créée en 1919 à l'école nationale des Beaux-Arts. Il forma de nombreux élèves tels que : Henri Marret (Paris, 1878 - Fourqueux, 1964), Louis Dussour (Riom, 1905 1986), Maurice Loutreuil (Montmirail, 1885 - Paris, 1925). Antoine Bourdelle (1861-1929), Marcel-Lenoir, son élève Louis Bouquet (1885-1952), le fils de Joseph Bernard (1866-1931) : Jean Bernard (1908-1994), René Piot (1866-1934), Costin Petresco (1872-1954, Professeur à l'académie des Beaux-Arts de Bucarest), Pierre Dionisi (1904-1976), Hector D'Espouy (1854-1929), sans oublier certains élèves des ateliers d'art sacré s'inscrivent à leur tour dans l'histoire de la fresque au XXe siècle. Paul Tournon confie par exemple la décoration des murs de son église du Saint-Esprit à Vincennes à une variété de fresquistes plus ou moins consacrés à cet art parmi lesquels il faut citer: Léon Toublanc $\left(\mathrm{XX}^{\mathrm{e}}\right)$, Pauline Peugniez (1890-1987), André Hubert Lemaître (1885-?), Lucien Weil (1902-1963), Raymond Pierre Virac (1892-1946), Valentine Reyre (1889-1943), Henri Charlier (1883-1975), Robert Pougheon (1886-1955), Odette Pauvert (1903-1966), Marthe Flandrin (1904-1987), Nicolas Untersteller (1900-1968), Élisabeth Chaplin (1890-1982)3. Rappelons toutefois l'influence de Puvis de Chavannes (1824-1898) qui emploie les tonalités adoucies de la fresque dans ses compositions murales monumentales et décoratives ${ }^{4}$, sur les 
fresquistes contemporains et Paul Baudoüin en particulier. Luce Barlangue considère que Marcel-Lenoir est l'initiateur avec Bourdelle de la rénovation de l'art mural ${ }^{5}$. Denis Lavalle et Jacques Thuillier dans leur article: «À propos de l'art mural en France » évoquent concernant l'art monumental: les «personnalités difficiles à classer (MarcelLenoir, Desvallières $\left.{ }^{6}\right) »$.

\section{Du désir de la monumentalité à la résurrection de la fresque au cœur du Quercy à Bruniquel}

3 Avant-guerre, précisément entre 1899 et 1914, Marcel-Lenoir élabore une série de projets d'œuvres, auxquelles le terme de "fresque» est rattaché: Chacun son Dieu (1911), L'Enfantement, la Vie et l'au-delà en 1899, La République c'est nous! en 1903, Sagesse en 1904, L'Ironie en 1908, La Famille mystique, La Décollation de saint Jean-Baptiste vers 1911-1914, La Mise au tombeau, La Crucifixion aux masques, en 1912. Marcel-Lenoir pour leur réalisation a recours à l'huile sur toile. Il s'agit alors d'une utilisation impropre du terme "fresque " pour désigner une peinture murale. Il est alors intéressant de souligner les encouragements de Puvis de Chavannes à Marcel-Lenoir, rapportés par René Ghil : « Puvis de Chavannes, lorsqu'il vit une enluminure de Marcel-Lenoir, la regarda longtemps, puis dit : « Bien. Cet ouvrier d'art commence par où un homme voué à l'art doit commencer ${ }^{7}$ ". Marcel-Lenoir admire notamment le Pauvre pêcheur ${ }^{8}$ de Puvis de Chavannes. L'exemple de ce maître le conforte dans des choix de composition synthétiques, le choix de couleurs adoucies, et le conduit vers l'épanouissement d'un art décoratif monumental sur toile, avant de se consacrer à la fresque. Dès 1899, l'enlumineur qui décore minutieusement la page d'un livre envisage déjà d'épouser l'art monumental pour ses projets: Chacun son Dieu, L'Enfantement, la Vie et l'au-delà. Le poète Émile Boissier remarque alors à propos de Chacun son Dieu: "Cette œuvre d'un symbolisme puissant dépasse les dimensions de l'enluminure. Aussi Lenoir l'exécutera-t-il en fresque, afin d'en mieux rendre toute l'intensité ${ }^{\prime}$. Un journaliste anonyme note :

« Enlumineur, Marcel-Lenoir devait être amené naturellement, comme ses ancêtres $\mathrm{du}$ quinzième siècle à la fresque. Et il nous donne la maquette d'une fresque pour la mairie de Montricoux : La République, c'est nous! (...) Marcel-Lenoir peut se faire une place remarquable dans la fresque et la peinture décorative des intérieurs d'édifices. (...) La fresque est l'équilibre harmonieux de la peinture, de l'architecture et de la conception philosophique qui guide l'ensemble ${ }^{10}$. » 


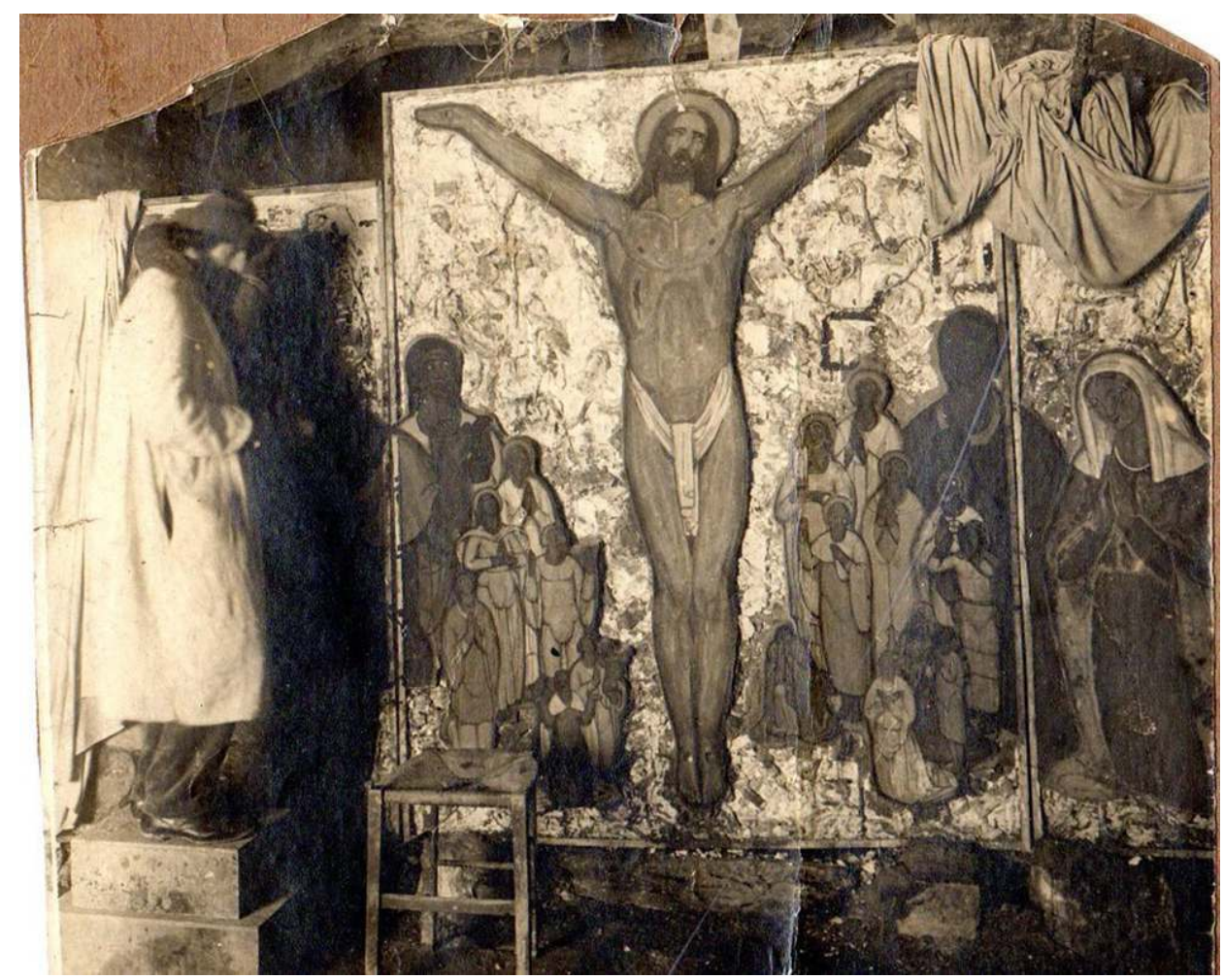

Photographie de Marcel-Lenoir devant la fresque : Le Christ au milieu des croyants et des incroyants à Bruniquel.

Photographe anonyme, vers 1917. () Archives Musée Marcel-Lenoir.

En 1913, Marcel-Lenoir vient pour la première fois à Bruniquel, commune médiévale du Quercy, et s'adonne à la fresque l'année suivante ${ }^{11}$. Cette commune, "florissante cité, renommée pour ses orfèvres. La noblesse des terres d'och y faisait ciseler ses joyaux ${ }^{12}$ " semble inspirer l'artiste. Il s'entoure d'artisans en bâtiment, de maçons, et loue une grange au bas du village afin de travailler à ses fresques de grandes dimensions (fig. $\mathbf{n}^{\circ} \mathbf{2}$ ). En 1916, six cimentiers commencent leur apprentissage de peintre comme les imagiers. Le maître exprime son souhait de réunir quinze ou trente ouvriers peintres, et envisage de fonder une école indépendante avec des élèves sans formation, mais prêts à tout apprendre ${ }^{13}$. Marcel-Lenoir termine 27 " peintures/fresques » à Bruniquel cette année-là 14. Marcel-Lenoir utilise une première technique sur ciment comme les artistes antiques, et dompte la matière en autodidacte. Jean Girou écrit :

«Pour travailler le "frésia", après une documentation historique, Marcel-Lenoir avait essayé de retrouver les veilles formules, les vieux matériaux; sur une armature de treillis de fer, il avait projeté un mortier de sable de Saint-Denis; il avait étudié le calfatage des péniches des mariniers; il a voulu atteindre aux procédés de la fresque primitive; tels étaient ses scrupules, sa conscience, son honnêteté15. "

Ces peintures monumentales des débuts sont composées de personnages en superpositions. D'épais cernes noirs suivent le contour modelé des personnages : ils sont en réalité les joints de séparation représentant les giornata. Le matériau est brut, épais, rugueux. Les visages et les membres sont tous d'une couleur ocre brun uniforme rehaussés de noir pour les traits. Les vêtements sont peints de couleurs pâles; blanc, 
jaune, orange, vert d'eau, et bleu léger. Les cheveux sont tous teintés de noir. Le fond est animé de paysages sombres et gris.

6 En 1920, le fresquiste change de technique, il enduit le mur d'un mortier composé de deux parties de sable, pour une partie de chaux. Afin de garantir sa solidité, l'artiste accroche à la main le mortier frais à une armature de treillage. Le rendu de ses œuvres est bien différent de ses premières peintures murales au ciment : la surface est uniformisée, lisse, les joints de séparation disparaissent au profit d'une harmonisation totale, d'une poésie et d'une douceur inégalée de tons frais, doux et pâles. Gaston Poulain décrit en 1926 cette fresque au mortier :

«Le mur est enduit d'un mortier formé de deux parties de sable pour une partie de chaux: sur cette préparation humide l'on peint avec de la poudre de couleur délayée d'eau. Dès que la poudre est incorporée, le silicate remonte à la surface et forme un vernis qui s'épaissit avec le temps et qui protège la fresque ainsi qu'une plaque de verre ${ }^{16}$.»

\section{Marcel-Lenoir et son Institut d'esthétique contemporaine : le visage oublié du professeur entouré de ses élèves}

7 En 1920, Marcel-Lenoir réalise son rêve et fonde l'Institut d'Esthétique Contemporaine dans son atelier, 115, rue Notre-Dame-Des-Champs. Ensuite, l'artiste emménage en 1928 au numéro 86 de cette même rue et devient le voisin de Fernand Léger. Dans le catalogue de l'exposition «Fernand Léger » en 1981, la plaque-enseigne intitulée : Institut d'esthétique contemporaine est remarquée, indiquant qu'il ne s'agit pas de Fernand Léger (1881-1955), mais ne précisant pas qu'elle désigne en réalité Marcel-Lenoir ${ }^{17}$. Roger Allard raille le « manifeste » où Marcel-Lenoir annonce l'ouverture de son institut. Il écrit : « Faisons à ce faux christ de cabaret montmartrois la publicité qu'il désire : Monsieur Marcel-Lenoir est fort bien embouti pour enseigner la fresque avec son abouti ${ }^{18}$ ». Le bois gravé du portrait de l'artiste par Chana Orloff (1888-1968), figure de son entourage, orne la première page de couverture (fig. $\mathbf{n}^{\circ} \mathbf{3}$ ). 
Figure 3

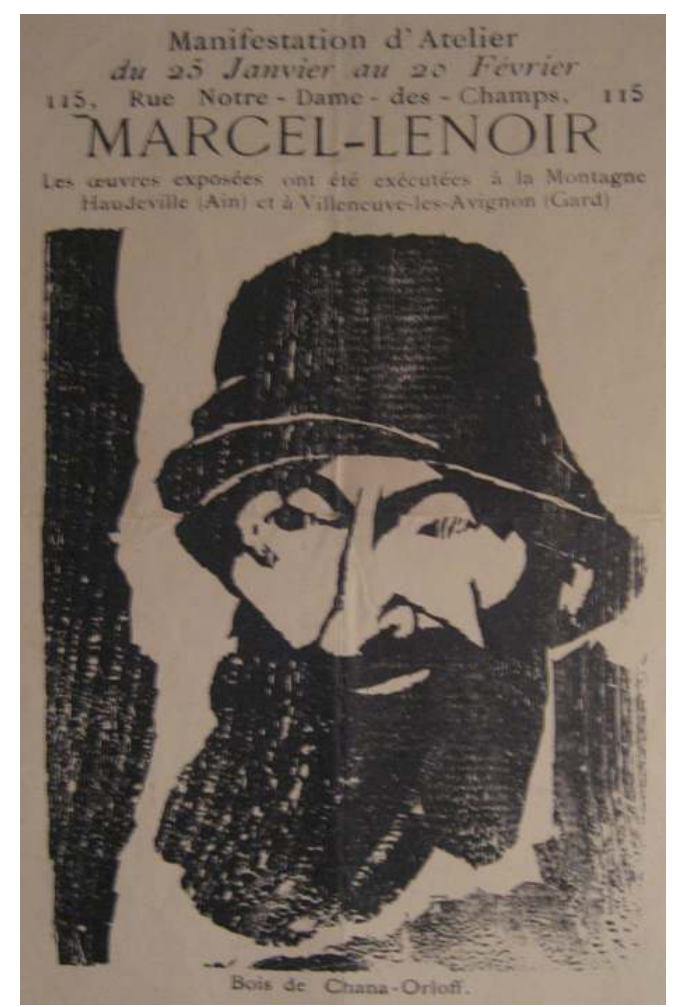

Première page de couverture du « manifeste » de la création de l'Institut d'Esthétique Contemporaine, 1920.

Phot. Namy, Jean-Baptiste, 2012. ㄷ) Archives Musée Marcel-Lenoir.

Marcel-Lenoir annonce l'ouverture de son école, son but, ses objectifs et ses conditions. Il entend enseigner la technique de la fresque : «Si des artistes se veulent instruire de la fresque, de sa technique et de son abouti, je consentirais volontiers à faire leur apprentissage, (...), ces artistes tout en faisant leur apprentissage, consentiraient à m'aider dans ma tâche ${ }^{19}$ ». À la suite de cours, d'évaluations, les élèves jugés dignes d'être associés à un travail en une collaboration de maitre à élèves, peindront directement sur le mur, et participeront aux différentes étapes qu'implique la réalisation d'une fresque. Marcel-Lenoir et ses élèves dessinent les croquis préparatoires pour l'œuvre future, avec la mise au carreau. Puis après de nombreuses études, lorsque celles-ci seront achevées, aux dimensions et proportions réelles du mur, ils le prépareront. Celui-ci écrit alors :

«Comment personnellement pourrais-je songer à instruire autrui de ce que je voudrais réellement savoir, ce que je veux amener à bien si possible c'est des collaborateurs qui n'auront qu'un unique but celui de s'incarner en ma production et de m'aider en agissant ainsi à plus m'exprimer ; aussi aurais-je recours à eux pour ce qui est de la préparation des matériaux indispensables à l'exécution des fresques et constamment je leur demanderai soit des études dessinées ou peintes, études qui seront pour moi des documents que je ramènerai, ce fatalement, à mon esthétique, et ils devront, quand nécessité sera, s'attaquer directement à la muraille $20 »$.

Marcel-Lenoir avertit les futurs élèves de la difficulté de l'art de la fresque :

«Je tiens à prévenir ceux qui pourraient venir à moi que souventes fois on est obligé de veiller très tard dans la nuit et que l'art de la fresque est un labeur vraiment pénible, non seulement cérébralement mais encore physiquement et 
enfin que le maniement de la chaux à la longue finit par occasionner des brûlures parfois douloureuses ». mois :

"Comme la fresque est œuvre d'amour, de passion, de dévouement je ne puis appeler à moi les faméliques ", " la fresque est un art vraiment onéreux et force m'est d'avoir recours aux artistes qui pourront me donner tout leur temps et m'aider financièrement: non que je désire en agissant ainsi tirer profit de cet argent ainsi apporté mais bien pour avoir la certitude que leur collaboration en ce que touche à l'appris de la fresque est toute de dévouement et de générosité ${ }^{21}$ ».

11 L'institut d'esthétique contemporaine rencontre un grand succès, et concrétise la renommée du fresquiste. Peu à peu, la figure de Marcel-Lenoir s'ajoute au nombre des maîtres avertis et expérimentés. Il s'impose, et compte alors parmi les personnalités les plus importantes dans la résurrection de la fresque. De nombreux élèves français mais également étrangers se dirigent vers l'atelier du maître. Nous pouvons affirmer la présence de : Pierre Claude Dubois $^{22}$, du franco-mexicain Jean Charlot ${ }^{23}$, du yougoslave Licenoski-Lasar ${ }^{24}$, des croates Jozo Kljakovic ${ }^{25}$, Ivo Resek ${ }^{26}$, du brésilien Antonio Gomide ${ }^{27}$. Jean Charlot exporte au Mexique les techniques de la peinture murale étudiées auprès de Maurice Denis (1870-1943), ainsi que de Marcel-Lenoir ${ }^{28}$. Serge Fauchereau écrit également :

« Jean Charlot fait partie de la Guilde Notre-Dame, un groupe de jeunes artistes sous l'influence de Maurice Denis, mais plutôt qu'à ce dernier, il vouera une reconnaissance durable à Marcel-Lenoir, artiste aujourd'hui oublié qui avait le mérite de défendre l'art mural qui déjà retenait $\operatorname{Charlot}^{29}$ ".

L'épouse de Licenoski-Lasar, Zoé, ancien conservateur du Musée national de Skopje, commente :

«Comme jeune artiste, mon mari a eu une bourse pour l'atelier Marcel-Lenoir. (...) Ce dernier lui a proposé de faire quelques dessins de composition, c'était un genre d'examen... Il ne prenait pas facilement des élèves. (...) Dans son atelier, se pressaient de grands personnages. Le gouvernement yougoslave considérait Marcel-Lenoir comme le meilleur fresquiste de Paris et du continent ${ }^{30}$. »

Licenoski-Lasar aide à la préparation des cartons de fresque pour l'Institut Catholique de Toulouse, puis en 1928, il collabore au projet de la fresque À la gloire de Dieu. À cette date, il réalise le portrait de Marcel-Lenoir en une huile sur toile. Marcel-Lenoir accompagne régulièrement son élève à $L a$ Coupole, et lui présente son entourage. Jozo Kljakovic devient également l'élève de Marcel-Lenoir en 1920. Giorgio de Chirico dans son ouvrage : Jozo Kliakovic publié en 1947 à Rome, souligne qu'il fut élève de Marcel-Lenoir, avec lequel il a appris l'art de la fresque ${ }^{31}$. Dans U suvremenom Kaosu : uspomene $i$ dozivljaji (Dans le chaos contemporain: souvenirs et expériences), Jozo Kljakovic précise à nouveau sa rencontre avec Marcel-Lenoir, et rappelle l'amitié et la collaboration de Marcel-Lenoir et Raymond Duncan, ainsi qu'une visite de Jozo Kljakovic en compagnie de Marcel-Lenoir dans l'atelier de Bourdelle ${ }^{32}$. Ivo Rezek étudie dans l'atelier de Derain, et acquiert les bases de la fresque auprès de Marcel-Lenoir, et en particulier, lors des contacts de ce dernier avec la Sorbonne. Antonio Gomide, après une convalescence à Genève afin de soigner sa tuberculose, rejoint Marcel-Lenoir à Toulouse en 1923, où il habite, et devient son élève. Il collabore au Couronnement de la Vierge à l'Institut Catholique de Toulouse ${ }^{33}$. Ce travail devra marquer la création de l'élève brésilien d'une empreinte ineffable; désormais ébloui par les fresques mystiques du maître, il s'investit à son tour dans le renouvellement de l'art sacré moderne en des images de la Figure du Christ (vers 1923), Descente de Croix (vers 1923), Sainte Cène (vers 1929). Toutefois, si Antonio Gomide rejoint 
Marcel-Lenoir sur un plan iconographique touchant au sacré, sur un plan esthétique, l'artiste brésilien utilise un vocabulaire spécifique de l'art déco.

L'institut d'esthétique contemporaine est assez comparable sur le plan du fonctionnement et de l'éthique envisagée aux Ateliers d'art sacré, on y sent pleinement l'esprit de corporation médiévale qui l'anime. Mais à la différence de certaines écoles privées contemporaines, Marcel-Lenoir en est le seul professeur. Une amitié fidèle se tisse entre Marcel-Lenoir et l'ensemble de ces personnalités autour de l'art et la fresque. Ainsi, collaborent à celle du Couronnement de la Vierge de l'Institut Catholique de Toulouse: Pierre Claude Dubois, Licenoski-Lasar, Antonio Gomide, mais également Louis Bouquet, et Maurice Albe ${ }^{34}$.

\section{Marcel-Lenoir : une esthétique moderne mystique, poétique et théâtrale...}

De la première technique, se détache la fresque du triptyque de la Crucifixion: Le Christ au milieu des croyants et des incroyants (Musée Marcel-Lenoir) vers 1917. La Vierge à l'enfant, travail préparatoire pour le panneau central, est exposée à la galerie de Goupil, rue de la Ville-L'évêque à Paris, et remporte un certain succès en $1917^{35}$. Banville d'Hostel s'écrie alors :

«Puis il emportera un souvenir élevé de la noble fresque de Marcel-Lenoir : c'est une scène savamment construite à traits viriles, dans une pâte solide : l'inspiration y allie la simplicité et la grandeur des formes aux sentiments les plus extatiques. La Vierge amplement drapée dans le pallium rappelle la grave matrone de l'époque romane ; la brune adorante qui se tient à sa droite est inoubliable. Cette œuvre est la révélation de ce salon ${ }^{36} »$.

Cette manifestation rassemble le groupe des Indépendants au bénéfice des œuvres de guerre. En outre, cette même année, Marcel-Lenoir réalise un ensemble de fresques de petites dimensions, portraits profanes et mystiques. Parmi ceux-ci, on peut signaler le Visage du Christ envoyé à l'exposition d'art français en 1917 à Barcelone (fig. $\mathbf{n}^{\circ} \mathbf{4}$ ), ainsi que la Vierge assise (répertoriée par Stanislas Fumet non localisée), L'Enfant à l'auréole exposé en 1917 à la galerie de Goupil, puis en 1919 à la galerie d'art des Éditions Grès et Cie, Paris (collection privée), et La Japonaise (collection privée). Ces œuvres, exécutées entre 1916 et 1917, présentent les mêmes caractéristiques stylistiques. 


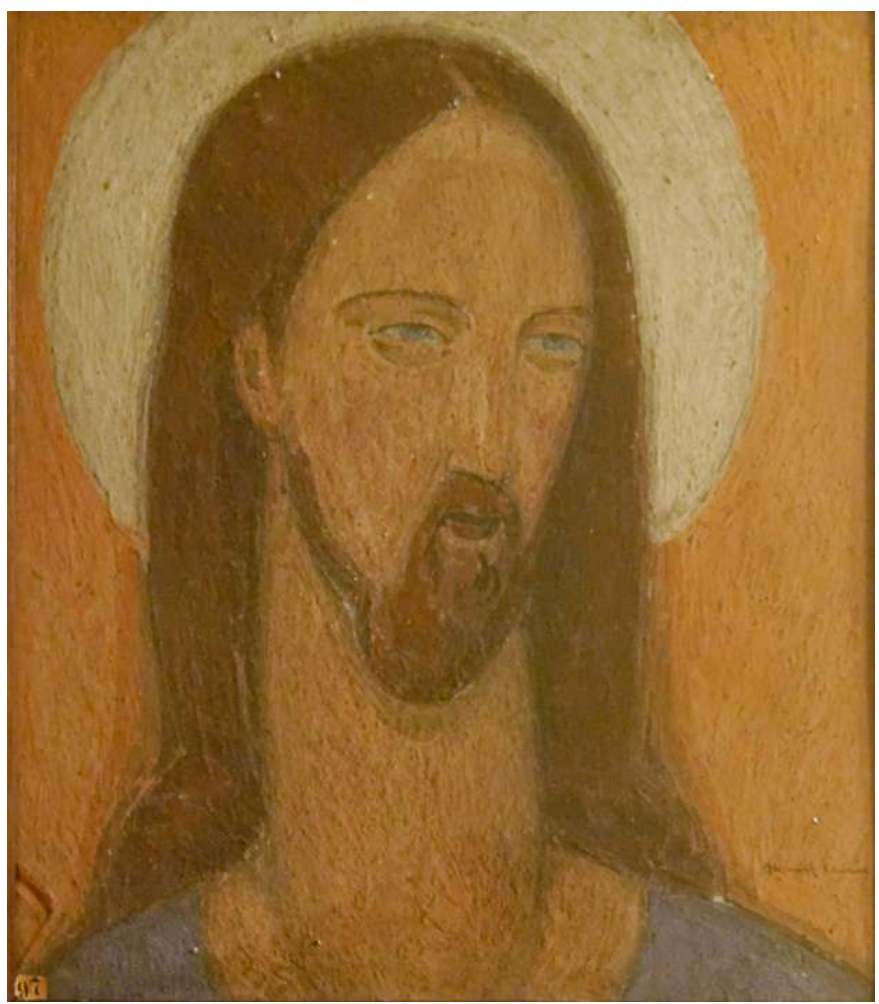

Marcel-Lenoir : Le Christ, fresque, vers 1917, collection particulière.

Phot. Namy, Jean-Baptiste, 2012. ( ) Archives Musée Marcel-Lenoir.

Vers 1917, Marcel-Lenoir réalise deux fresques de petites dimensions : Femme au ruban rouge, exposée à la galerie de Goupil en 1917, acquise par le ministre des Beaux-arts Monsieur Dujardin-Beaumetz le 26 mai 1917 pour le Musée du Luxembourg (Musée national d'art moderne du Centre Georges Pompidou), ainsi que La Vierge au voile bleu (Musée Marcel-Lenoir) (fig. $\mathbf{n}^{\circ}$ 5). La grammaire plastique moderne de l'artiste est mise au service de son mysticisme. La même jeune femme pose pour ces deux œuvres, qui constituent des esquisses préparatoires au panneau de droite du triptyque de la Crucifixion : le Christ au milieu des croyants et des incroyants. L'artiste simplifie les traits de la Vierge. L'expression paisible et sereine de leurs visages évoque la pureté mariale. Elles inclinent légèrement leurs visages de trois-quarts. Dans Femme au ruban rouge, le regard à demi-clos s'unit à la gestuelle des mains jointes en signe de prière. Le parti pris de leurs visages recueillis et songeurs à travers un découpage anatomique très plastique, laisse transparaître l'admiration du peintre pour les fresques romanes, les vierges et les anges de Cimabue (1240-1302). L'ovale des visages est souligné par des arcades sourcilières appuyées, des paupières à demi-closes, des lèvres douces teintées de carmin. Le style adopté confère à ces œuvres un sentiment de noblesse et de paix. 
Figure 5

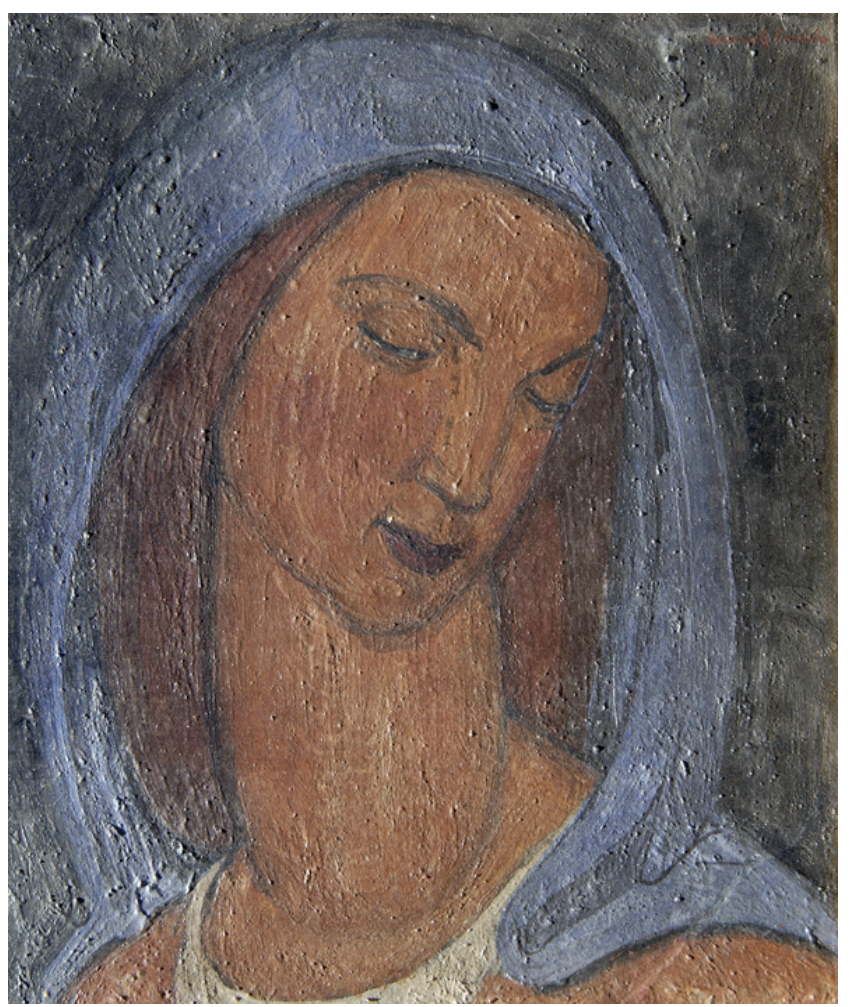

Marcel-Lenoir : Vierge au voile bleu, fresque, vers 1917. Musée Marcel-Lenoir.

Phot. Namy, Jean-Baptiste, 2011. ( ) Archives Musée Marcel-Lenoir.

En 1918, rappelons l'exécution de la commande à fresque au ciment pour la décoration du tympan de la façade de l'hôtel particulier de Louise Lara et d'Édouard Autant-Lara, situé 2, rue Émile-Ménier. Louise Lara est une comédienne avant-gardiste, syndicaliste militante, liée d'abord à Paul Fort et Lugné-Poe, puis de 1899 à 1919, elle entre à la Comédie-Française. Édouard Autant est architecte, anarchiste, anticlérical, et socialiste. Ensemble ils fondent le groupe Art et Liberté en 1917, qui devient le laboratoire Art et Action en 1919. Ce théâtre d'avant-garde et de recherche a pour objectif de faire connaître de l'inédit, vise la quête de l'unité, la simplification symboliste et soutient le futurisme. Ce réseau d'amitiés: Barzun, Divoire, Voirol, Marinetti, Apollinaire, Polti, Dujardin, Larronde, Mercereau, Gazanier, Roisnard qui composent le Comité de Fondation d'Art et d'Action collabore "pour la défense des œuvres modernes" sur un front poétique symboliste avec pour modèle René Ghil ${ }^{37}$. Marcel-Lenoir adapte sa décoration à l'architecture. L'artiste évoque un univers idéal, poétique et symboliste, adoptant une esthétique moderne, des formes rondes, robustes, puissantes. La conception à la fois éthique et esthétique de cette décoration rejoint les idées chères et défendues par les commanditaires. De chaque côté de l'entrée se tiennent, à gauche, Édouard Autant reconnaissable à ses instruments d'architecte, à droite, Louise Lara tenant son enfant sur les genoux. Au centre, triomphe le bonheur de leur union en leurs visages réunis, tandis qu'au-dessus d'eux plane une déesse avec le baiser de deux colombes ${ }^{38}$. Le Musée Rodin ne conserve aucune trace d'archive, en outre, ce bâtiment a été détruit. Cependant, il subsiste une photographie dédicacée: "De tout cœur à l'admirable et sérieux artiste, dévoué aux Arts, Autant Lara, avril $1921^{39}$ » (fig. $\mathbf{n}^{\circ} \mathbf{6}$ ). Rodin collabore également à ce projet avec sa sculpture du Désespoir, Chana Orloff avec un bois animalier, Charles Despiau 
(1874-1946) avec un médaillon ${ }^{40}$. Cette commande souligne la présence de Marcel-Lenoir au cœur du paysage artistique, théâtral et littéraire à cette période.

Figure 6

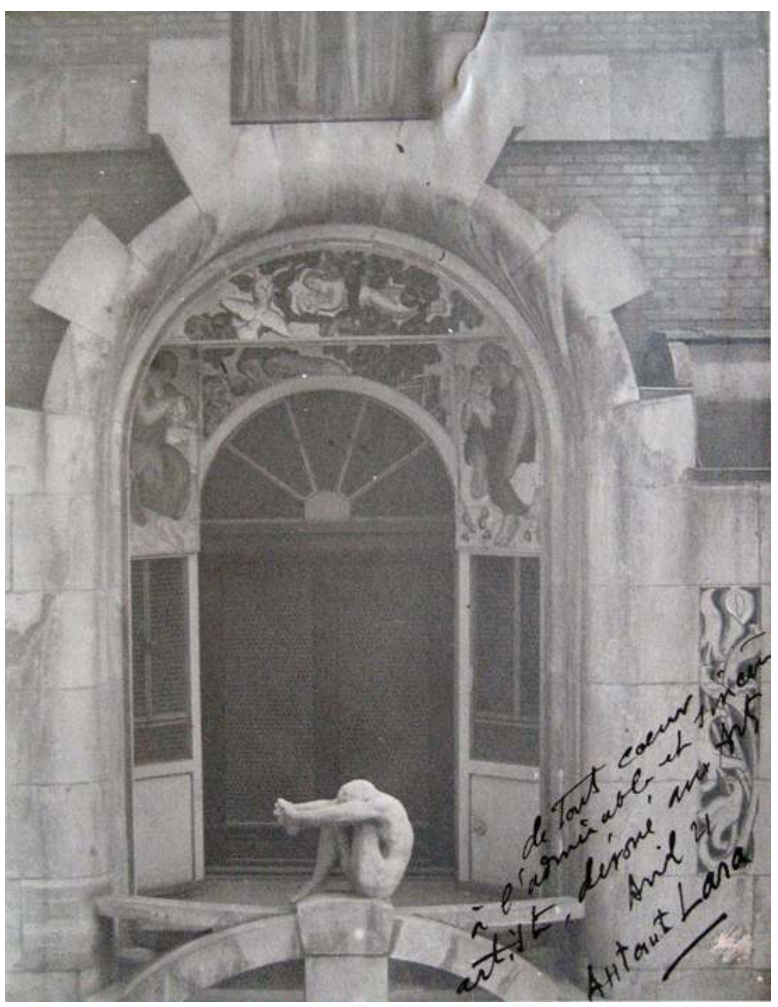

Photographie dédicacée à Marcel-Lenoir par Édouard Autant en 1921.

Photographe anonyme. (c) Archives Musée Marcel-Lenoir 


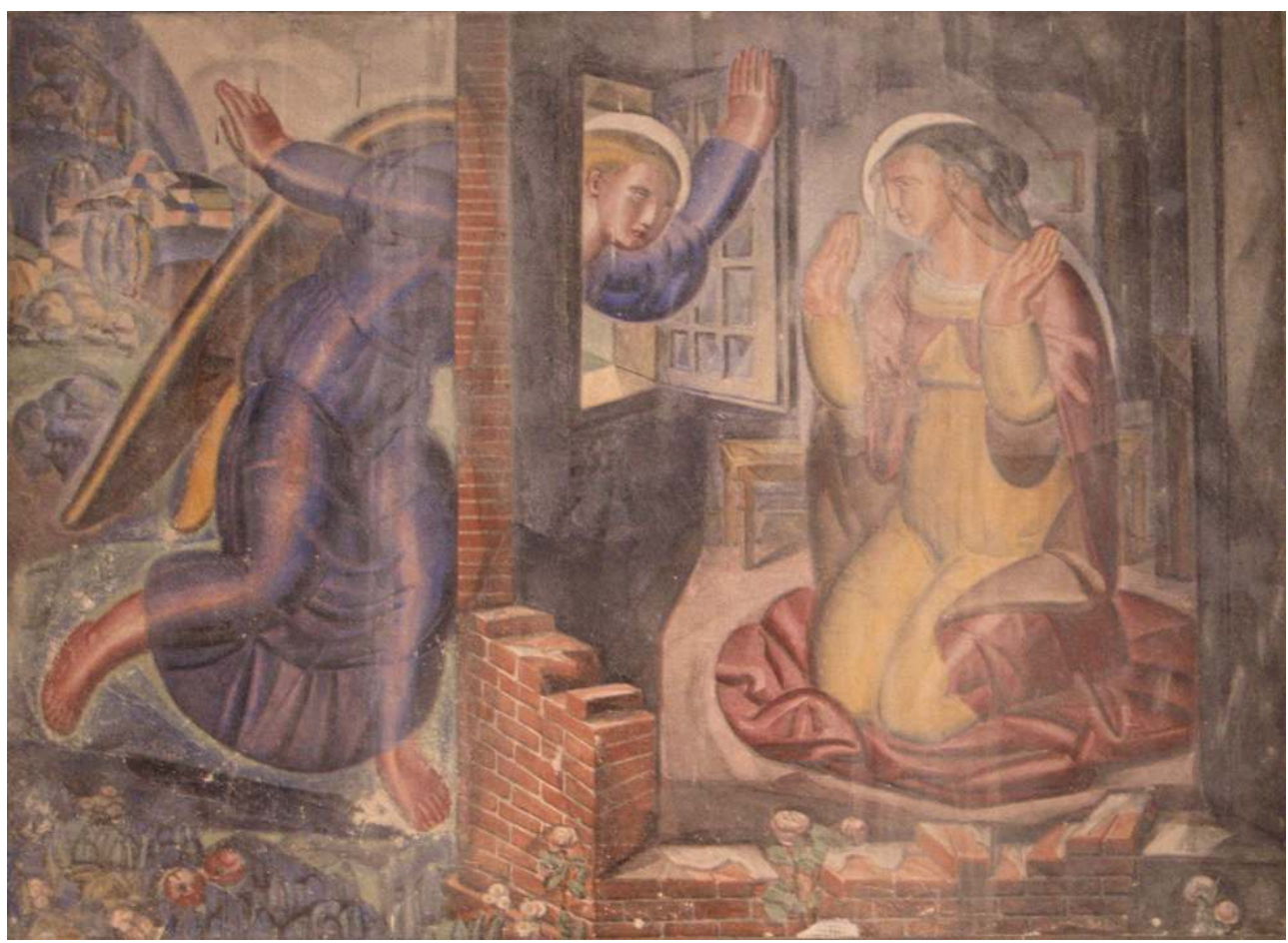

Marcel-Lenoir : L'annonciation, fresque, vers 1920. Montricoux, Église Saint-Pierre.

Phot. Namy, Jean-Baptiste, 2012. @ Jean-Baptiste Namy.

L'Annonciation scellée dans le chœur à gauche dans l'église de Montricoux est l'une des premières fresques où Marcel-Lenoir utilise son nouveau procédé (fig. $n^{\circ} 7$ ). Elle n'est pas datée, mais est exposée au Salon d'Automne en $1920^{41}$ où elle remporte un grand succès ${ }^{42}$. Elle est présentée également à la Galerie Adolphe Le Goupy, puis à la Sorbonne, où l'écrivain et critique d'art Guy-Félix Fontenaille (1892-1985) organise une conférence sur $l^{\prime} œ u v r{ }^{43}$. Lignes, ouvrage lyonnais reproduit deux fusains préparatoires à l'Annonciation ${ }^{44}$. L'artiste renouvelle ce thème popularisé à la fin du XIII ${ }^{e}$ siècle par Jacques de Voragine. Marcel-Lenoir dépouille l'iconographie de plusieurs attributs, afin de se concentrer sur l'essentiel à partir du geste, symbolique et fondamental dans toute l'œuvre de l'artiste. Dans un parti renaissant, il ouvre la perspective sur un paysage vallonné du Quercy dont le premier plan de verdure est parsemé de roses et de pâquerettes, fleurs mariales. La Vierge est agenouillée dans une humble maison de briques toulousaines au cœur du village. Vêtue d'une robe d'or, et d'un manteau rouge, couleurs de royauté, les mains sont levées en signe d'approbation de la volonté de Dieu et de prière contemplative. Sa position à même le sol montre son humanité, son rattachement à la terre, pour celle qui va devenir mère de Dieu. Ici, Marcel-Lenoir a choisi la conturbatio, le trouble, la surprise, et en même temps sa crainte, que nous pouvons rapprocher des cinq états mentaux et spirituels de la Vierge au moment des paroles de l'Ange, définis par le prédicateur franciscain Roberto Caracciolo. Elle est placée à droite, symbole de justice signifiant la bienveillance, la miséricorde divine, dans un intérieur dont l'architecture est un claustra ouvert. La verticalité du mur peut représenter l'arbre de vie, le symbole du Christ, et crée l'union du ciel et de la terre. L'ange qui appartient au monde de l'au-delà, surgit dans son envol, il incarne l'«action » et la surprise au sein du mystère de l'Annonciation. Il apparait à la fenêtre ouverte, celle-ci laisse pénétrer un rayon de lumière, emblème divin, 
en une diagonale descendante signifiant l'incarnation de Dieu. En une modernité de conception, l'artiste utilise des solutions de détails apparentées au cubisme, une simplicité de moyens, ainsi qu'une dynamique du rythme, proche de l'esprit futuriste qui anime encore les années 1920. Les couleurs choisies par Marcel-Lenoir trahissent un esprit hautement inspiré, maîtrisant la symbolique des couleurs, science spirituelle, et communiant avec la langue divine et sacrée ${ }^{45}$. Cette œuvre n'est pas dans la lignée de l'art sulpicien, ni dans l'anecdote, elle est dans l'intemporalité. Dans leurs comptes rendus à propos du Salon d'Automne, L'Annonciation est remarquée et appréciée de différents critiques d'art tels que : Étienne Bricon qui parle d'une " témérité heureuse " ${ }^{46}$, Léon Plée cite L'Annonciation" ${ }^{47}$, André Gybal : «Marcel-Lenoir est comme un primitif, qui aurait vu des cubistes et n'aurait rien perdu à les fréquenter ; en sorte que sa fresque L'Annonciation est à la fois naïve et très construite : plaisant contraste ${ }^{48} »$.

Figure 8

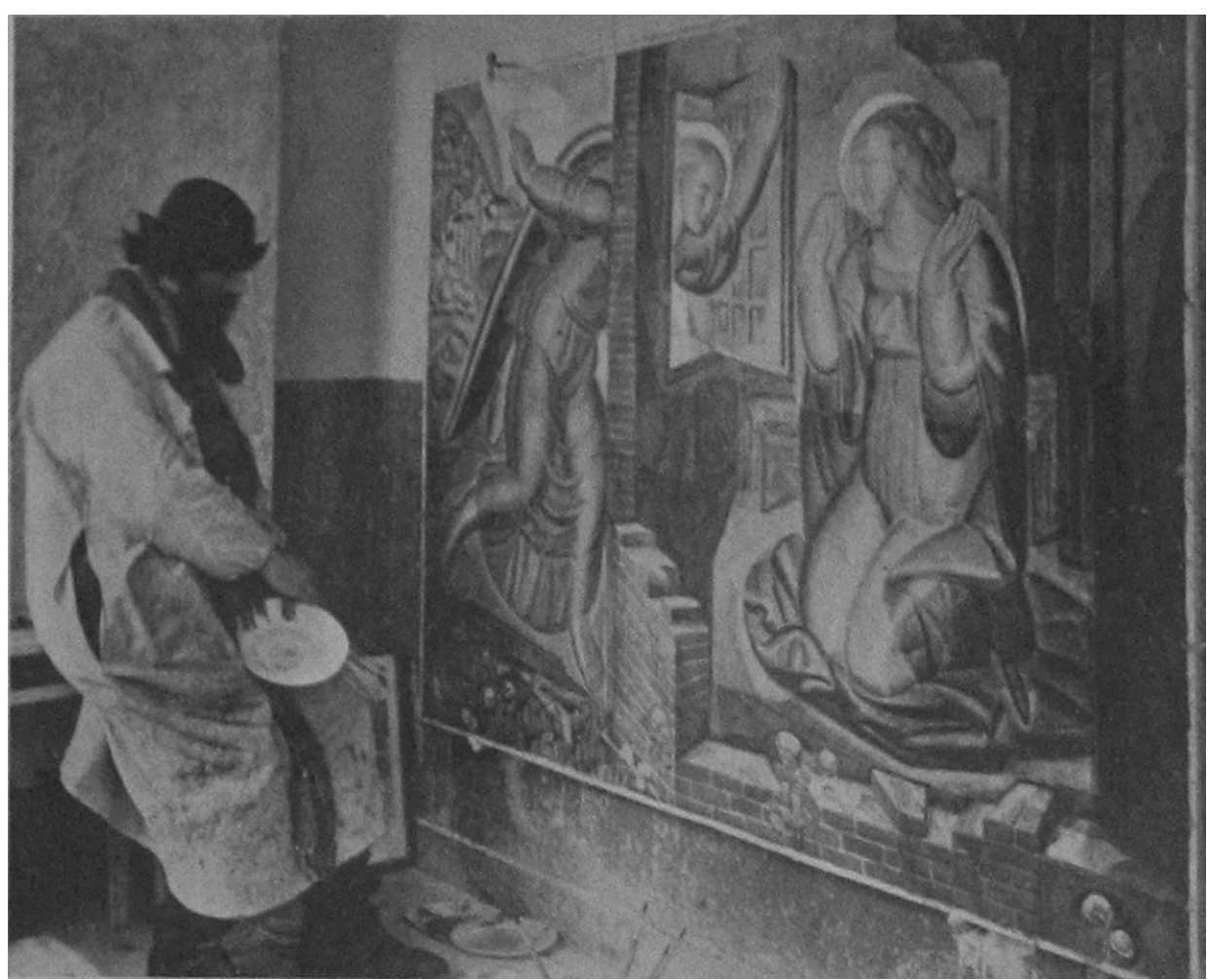

Photographie de Marcel-Lenoir dans son atelier parisien devant L'annonciation vers 1920.

Phot. Fister, P., vers 1920. (c) Archives Musée Marcel-Lenoir.

Grâce au fonds de plusieurs lettres conservées dans les archives municipales, sont apportées de nombreuses indications quant au don, à l'envoi, à la mise en place de la fresque (fig. $\mathbf{n}^{\circ} \mathbf{8}$ ). En 1922, Marcel-Lenoir fait don à la commune de Montricoux de L'Annonciation à condition qu'elle soit placée dans l'église dans le chœur au-dessus de la petite porte de la sacristie à gauche. Il exprime sa joie devant l'empressement du maire et du conseil municipal à accepter ce don. Marcel-Lenoir évoque le surcroît de dépenses dues à l'emballage car il doit abandonner une assurance trop onéreuse et renforcer la caisse par une assise solide avec l'aide de trois manutentionnaires afin de prendre toutes les précautions nécessaires pour ce transport délicat ${ }^{49}$. Lors d'une séance publique du conseil municipal du 18 février 1923, la commune accepte le don de Marcel-Lenoir pour 
l'église de la paroisse ${ }^{50}$. Le transport de la fresque est effectué de Paris par la société de Monsieur Frelier, et suscite de nombreuses discussions quant à l'emballage et aux coûts. Une souscription pour les frais occasionnés par l'envoi et la mise en place de l'œuvre sera décidée le 23 février 1923 en conseil municipal et comprendra la participation de 96 personnes de la commune ${ }^{51}$. L'abbé Pierre Augé, curé de l'église Saint Pierre de Montricoux reçoit la fresque le 23 décembre 1923 à la gare des chemins de fer de Montricoux. Il évoque les qualités, la renommée de Marcel-Lenoir en tant que grand artiste religieux. L'œuvre a été mise en place par le charpentier de Montricoux Monsieur Antonin Roux, elle est fixée comme l'a souhaité Marcel-Lenoir avec l'accord du père Augé dans le chœur de l'église, au-dessus de la porte de la sacristie ${ }^{52}$.

Figure 9

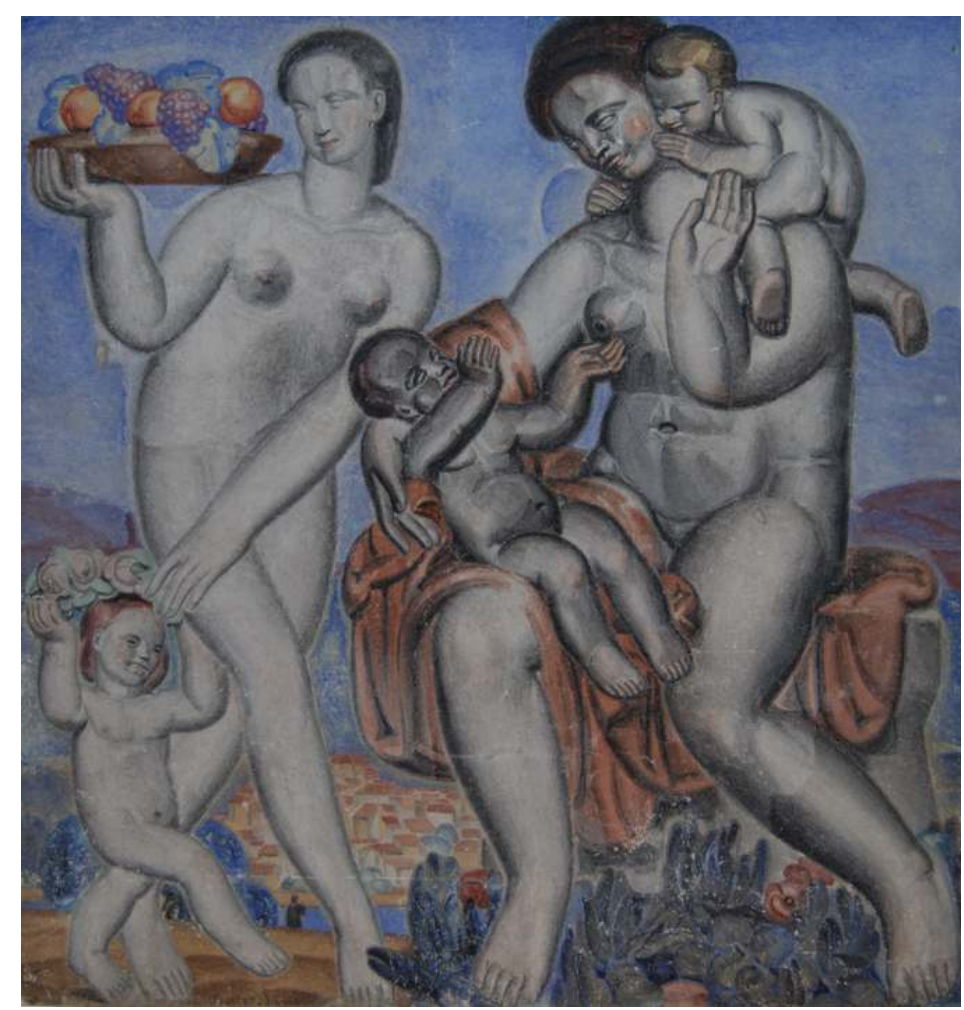

Marcel-Lenoir : Joie de vivre, fresque, vers 1921. Musée Marcel-Lenoir.

Phot. Namy, Jean-Baptiste, 2012. @ A Archives Musée Marcel-Lenoir.

En 1921, Marcel-Lenoir expose deux Joie de vivre et la Flagellation (Musée Marcel-Lenoir) au Salon d'Automne ${ }^{53}$, ainsi que l'une d'elle à la Société Nationale. Plusieurs critiques d'art les remarquent et les citent dans leurs comptes rendus de la Nationale : Vanderpyl ${ }^{54}$, ainsi que Georges Denoinville ${ }^{55}$. Pour la Joie de vivre (fig. $\mathbf{n}^{\circ} \mathbf{9}$ ), Marcel-Lenoir célèbre la terre nourricière dans ce paysage ensoleillé du Midi toulousain, la richesse de cette région bienfaitrice sous forme de corbeilles regorgeants de fruits et de fleurs, et du bonheur des deux mères nourricières à travers les deux maternités. Celle de gauche danse avec son enfant. Elle rappelle l'œuvre de son ami inséparable Joseph Bernard, notamment Femme à l'enfant de 1914, le haut relief en pierre de Lens de 1918: Maternité, mais également les Bacchantes de $1919^{56}$. Celle de droite, de couleur noire, nous fait songer au modèle célèbre de Montparnasse : Aicha avec laquelle Marcel-Lenoir est $\mathrm{ami}^{57}$. Elle porte sur ses épaules un enfant se blottissant contre son cou, ainsi qu'un second bébé assis sur ses genoux, 
réclamant le sein de sa mère avec un geste d'imploration. La composition en forme de croix est structurée par les diagonales formées par les deux jeunes femmes. Celles-ci semblent être des blocs inébranlables. Les volumes sont dessinés en douceur, en lignes souples, et traduisent également une composition synthétique semblable aux sculptures d'Aristide Maillol (1861-1944), Joseph Bernard, Charles Despiau, Robert Wlérick (1882-1944), Henry Parayre (1879-1970), ou même André Abbal (1876-1953). Dans un camaïeu de gris bleuté, d'indigo, et de carmin, Marcel-Lenoir met en lumière l'union de la mère et de l'enfant, l'harmonie de cette communion entre l'homme et la nature. La seconde Joie de vivre est symbolisée par la danse (fig. $\left.\mathbf{n}^{\circ} \mathbf{1 0}\right)$, et incarne également l'éloge de la Création. Autour de ces œuvres de grandes dimensions, Marcel-Lenoir réalise également Les Trois Visages (datés 1920-1921).

Figure 10

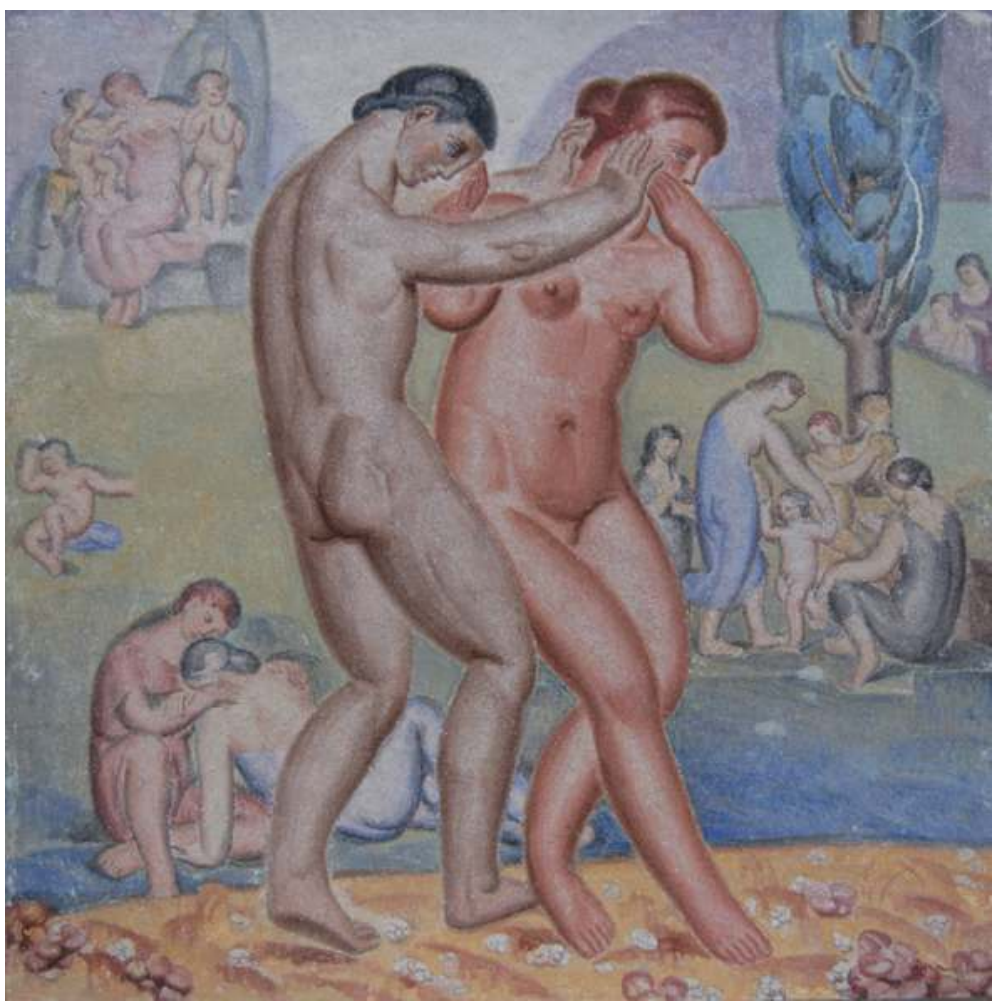

Marcel-Lenoir : Joie de vivre, fresque, vers 1921. Musée Marcel-Lenoir.

Phot. Namy, Jean-Baptiste, 2012. ( ) Archives Musée Marcel-Lenoir.

Dans les années 1920-1921, Marcel-Lenoir s'adonne à la réalisation de fresques de petites et de moyennes dimensions, parmi lesquelles : Tête de femme (collection privée), Visage de jeune femme (monogrammée, 1920, collection privée), $N u$ debout (1920, collection privée), Tête de jeune homme (1921, collection privée), Madeleine (1921, collection privée), Nu allongé (1921, collection privée), Danse (1921, collection privée), Scène pastorale à la guitare (1921, collection privée), Jeune fille aux anglaises (1921, Musée Marcel-Lenoir), Autoportrait (1921, collection particulière). Il existe une correspondance, une harmonie entre celles-ci et les peintures de cette même époque. Il expose lors d'une manifestation d'atelier au 115, rue Notre-Dame-des-Champs en 1921 un ensemble de fresques: Femme au corsage rouge, Pastorale, La Petite Source, Femme à l'écharpe bleue, Portrait de l'auteur, La Flagellation, Tête de fermme, Femme à la corbeille, Femme au bras levé, Candeur, Tête de Vierge, Femmes aux bras levés, 
Jésus au jardin des oliviers, La Joie de vivre, Tête de fermme, La Femme aux yeux mi-clos, Femme aux anglaises (fig. $\mathbf{n}^{\circ} \mathbf{1 1}$ ), Joie de vivre, Les Fruits, Sanguine, La Jolie Maison, Ails et noix.

Figure 11

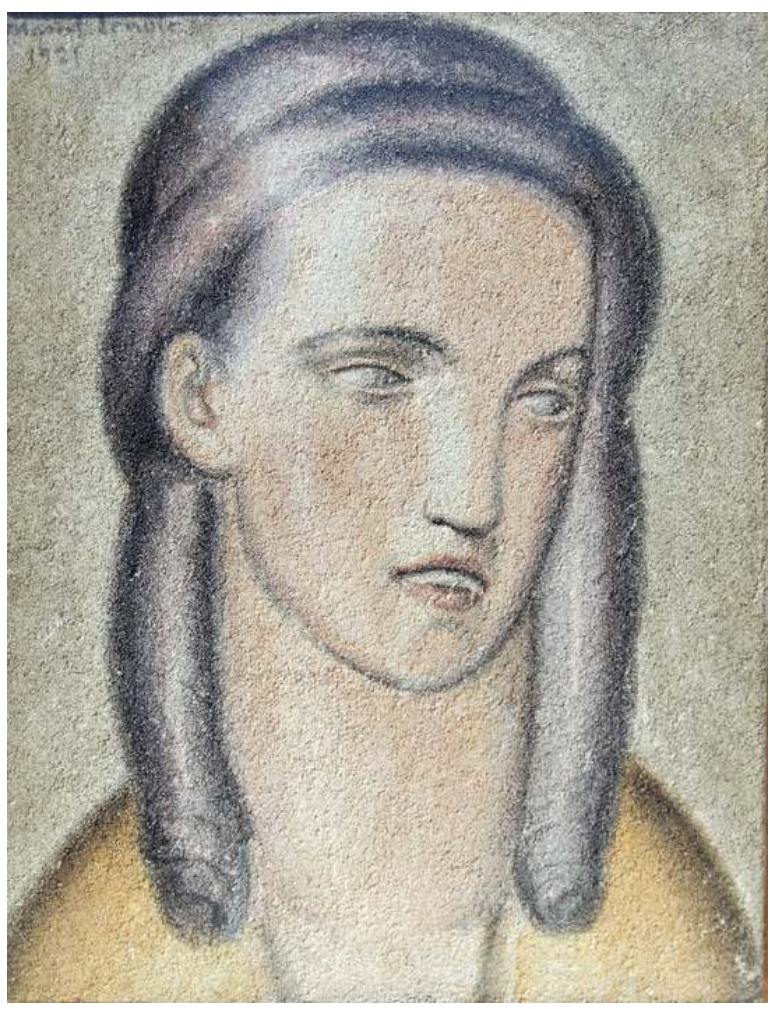

Marcel-Lenoir : Femme aux anglaises, fresque, 1921. Musée Marcel-Lenoir.

Phot. Namy, Jean-Baptiste, 2011. ( ) Archives Musée Marcel-Lenoir.

L'Autoportrait ou Portrait de l'auteur est daté de 1921 (fig. n¹2). L'artiste se représente en buste, de face, paré de son chapeau de feutre noir, et vêtu de sa blouse blanche de fresquiste. Il restreint sa palette en des tonalités claires de vert, de blanc, d'ocre accompagné d'un parti-pris synthétiste. Son image est conforme aux photographies d'époque de Marc Vaux, Vizzanova, Choumoff, comme l'artiste ténébreux à la fois obscur, légendaire, mythique et immortel, scrutant le spectateur de son regard d'aigle intransigeant. Ici, Marcel-Lenoir incarne son rôle d'initiateur, de mage, de maître et inaugure également l'artiste-héros, qui nous semble se rapprocher de Thomas Carlyle dans son Culte des héros. Rappelons la description d'André Salmon à son propos : «L'un des plus fichus caractères d'aucun temps et d'aucun pays ${ }^{58}$ ». 


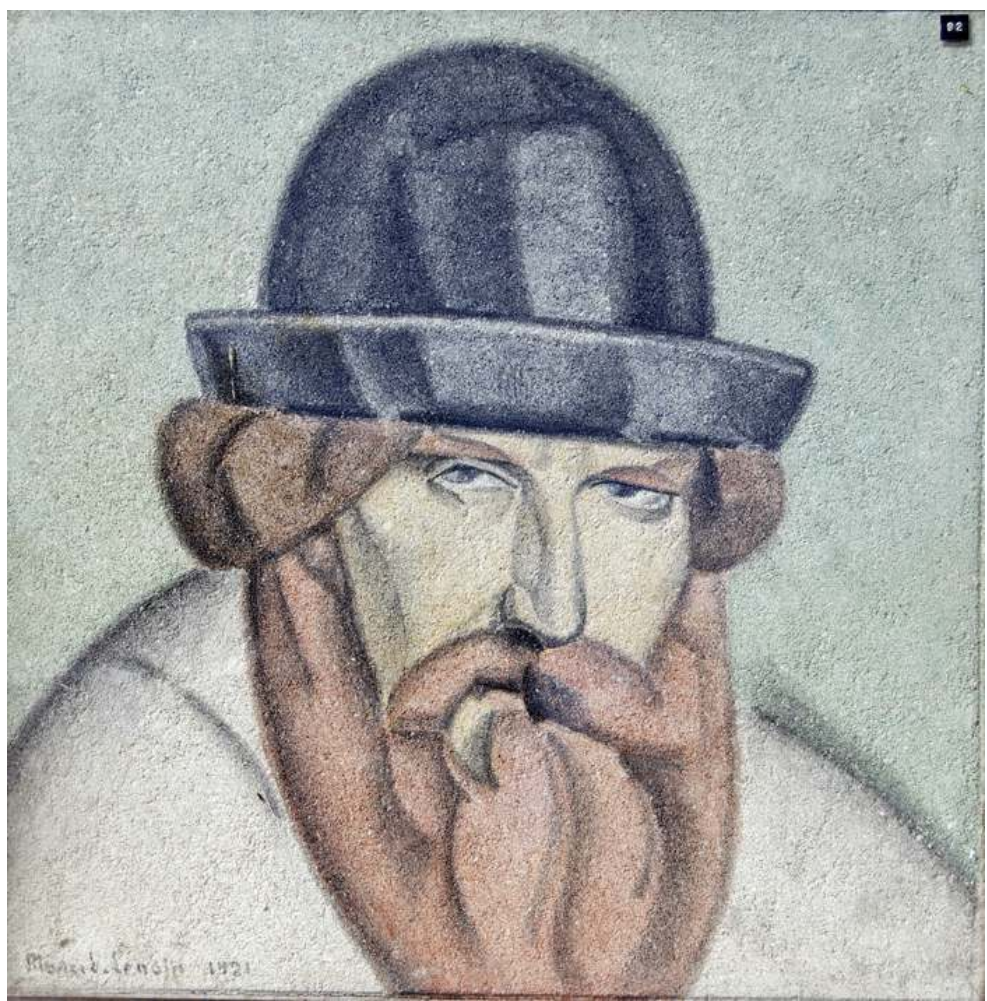

Marcel-Lenoir : Portrait de l'auteur, fresque, 1921. Collection particulière.

Phot. Namy, Jean-Baptiste, 2011. ( ) Archives Musée Marcel-Lenoir.

$\mathrm{Au}$ sein du legs de 125 œuvres fondateur du Musée Marcel-Lenoir à Ribeauvillé, nous recensons plusieurs fresques : La Femme aux anglaises, Tête de Christ, Flagellation, Les Heureux , ainsi qu'une « grande fresque » sont présentées.

Figure 13

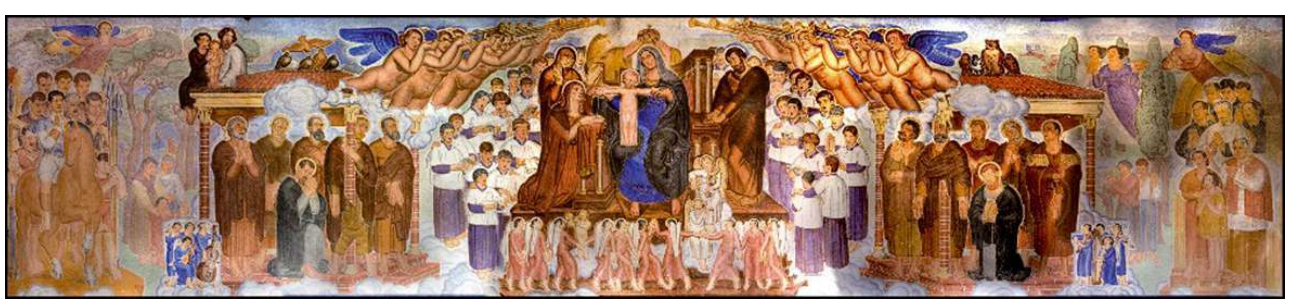

Le Couronnement de la Vierge, fresque, 1920-1923. Institut Catholique de Toulouse.

Phot. Bouquillon, Gilles. (c) Archives Musée Marcel-Lenoir.

Entre 1920 et 1923, l'artiste réalise la commande du Couronnement de la Vierge (fig. nº13) pour l'Institut catholique de Toulouse dirigé par le recteur Monseigneur Breton, sous la recommandation de Camille Soula qui déclare: «C'est bien un spectacle qu'il faut dire pour distinguer cette peinture de toutes les peintures et marquer tout ce que la matièrefresque apporte de vie supérieure à cette fête éternelle ${ }^{59}$ ». Madame le professeur Luce Rivet fut la première à s'intéresser à cette œuvre : «L'exécution de la fresque de MarcelLenoir à l'Institut Catholique fut considérée par les amateurs comme un temps fort de la création artistique de "l'entre-deux-guerres" à Toulouse ${ }^{60}$. " En visionnaire, il renouvelle 
l'iconographie de ce thème, réinterprétant les textes de La Légende dorée de Jacques de Voragine, La Louange de la Vierge Mère de Bernard de Clairvaux, le Psautier à la Sainte-Vierge de saint Bonaventure. Il en donne une représentation très novatrice, résolument moderne et profondément mystique. Il réunit la Vierge, le Christ, et Dieu le Père, dont le visage disparaît sous une source triangulaire lumineuse incandescente, en référence à l'idée théologique selon laquelle Dieu est lumière. L'artiste conjugue son admiration pour les Primitifs, Ingres, le cubisme qu'il traite de façon personnelle, et une fascination pour la sculpture qui se traduit par la plastique des corps puissants semblables à des blocs inébranlables. Il met à l'honneur autour du triangle symbolique les personnalités toulousaines qu'il considère comme les lumières intellectuelles de la ville : Camille Soula, le comte Henri Bégouen, Henri de Lingua de Saint-Blanquat, Gabriel du Bourg de Luzençon, Joseph Marie Xavier de Sévin, Jacques Adrien Crouzel, Mgr Breton, Mgr Germain, Mgr Thomas. Dans les années 1920, le Couronnement de la Vierge très singulier par son iconographie et son style est très commenté. En effet, Camille Soula, Stanislas Fumet, Jean-Louis Vaudoyer, Maurice Brillant, en font l'éloge ${ }^{61}$. À Toulouse, Marcel-Lenoir incarne une image possible de modernité, mais celle-ci ne fut pas comprise par tous, et la fresque suscita des passions dans la presse locale entre la désapprobation des uns et l'admiration des autres. Pour Gaston Poulain, il ne fait aucun doute : «L'art catholique de notre siècle n'a pas de pareil peintre ${ }^{62} »$. L'harmonie de la fresque en une symphonie colorée d'or, de rose, de rouge brique, d'ocre, et de violet, teintes prismatiques, incarne sur terre le paradis mystique de Marcel-Lenoir : Toulouse, qu'il érige en ville céleste. Le mystère glorieux proclamé par Marcel-Lenoir, par la profondeur de son élévation chrétienne, la grandeur de son idéal visionnaire, sa suprême religion esthétique le distingue alors de ses contemporains.

Figure 14

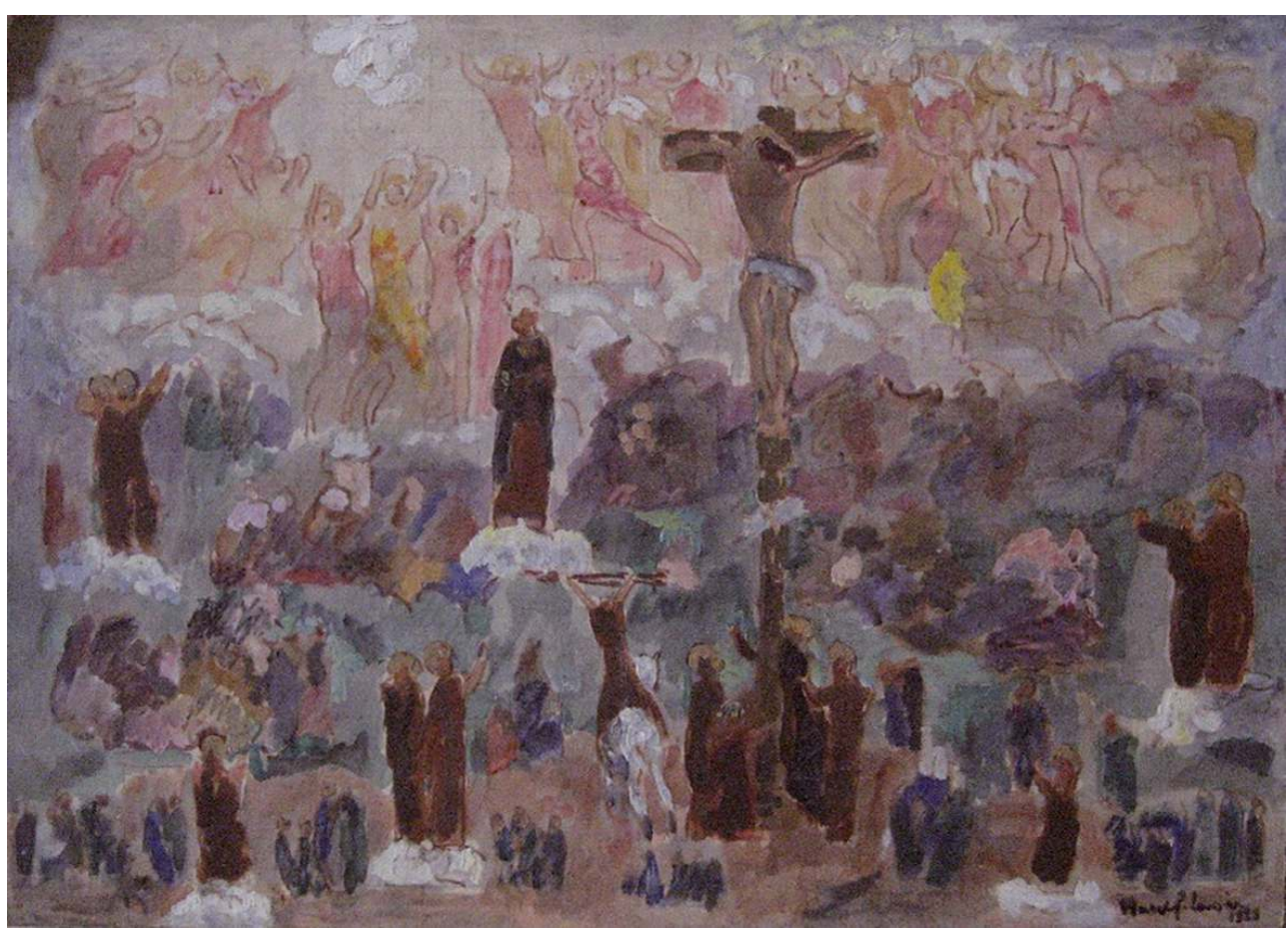

Marcel-Lenoir : À la gloire de Dieu, aquarelle et gouache sur panneau de bois, vers 1928. Musée MarcelLenoir.

Phot. Namy, Jean-Baptiste, 2012. (c) Archives Musée Marcel-Lenoir. 


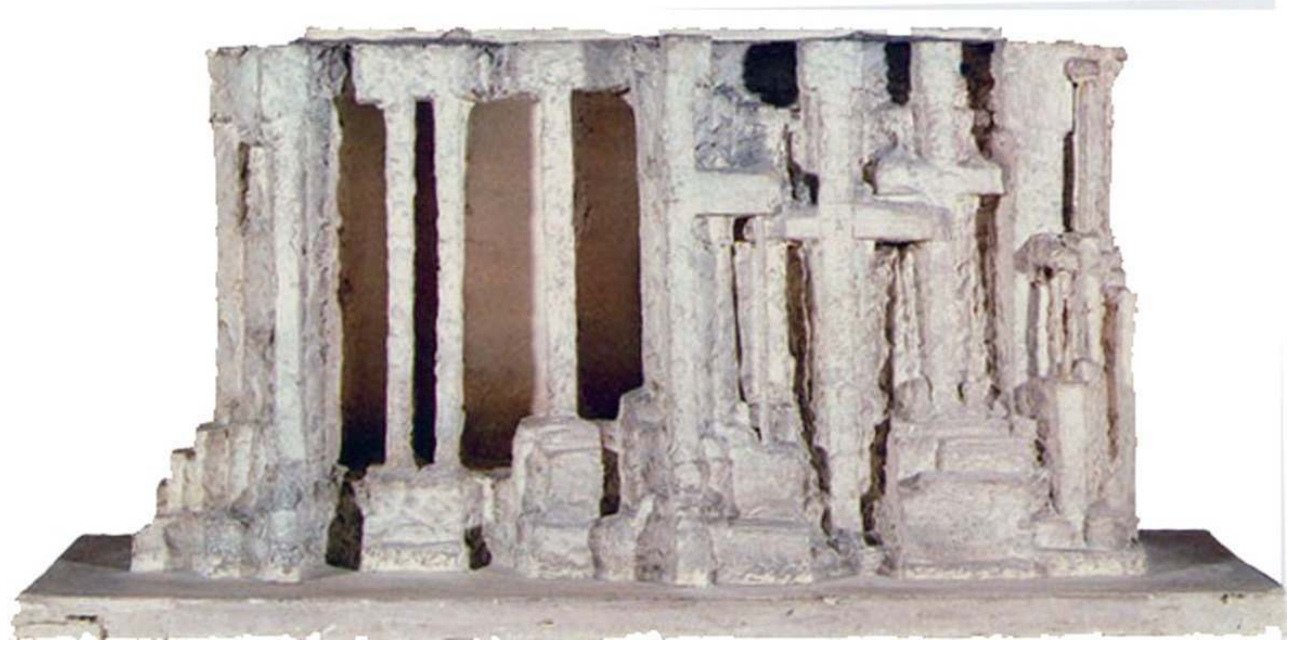

Marcel-Lenoir : À la gloire de Dieu, maquette en plâtre, vers 1928. Musée Marcel-Lenoir.

Phot. Namy, Jean-Baptiste, 2012. (c) Archives Musée Marcel-Lenoir.

La Gloire de Dieu (fig. $\mathbf{n}^{\circ} \mathbf{1 4}$ ) est la dernière fresque commencée par Marcel-Lenoir, projet monumental de $600 \mathrm{~m}^{2}$, qu'il souhaite ériger à Paris, au Trocadéro. Il réalise la maquette architecturée en plâtre (fig. $\mathbf{n}^{\circ} \mathbf{1 5}$ ) ainsi que plusieurs dessins préparatoires (Musée Marcel-Lenoir). Dans l'un de ses manifestes publiés, il écrit :

«Affronter un labeur de cette importance, c'est quintupler sa puissance créatrice, que dis-je, c'est la décupler ; c'est être victorieux sur ces propres défaillances, c'est exalter dans le calme enivrant des nuits ses possibilités-œuvrer, c'est s'éclairer d'une lumière que les craintifs ignoreront toujours, c'est utiliser à tous les instants les richesses lentement arrachées à la nature, c'est rendre à l'homme les bienfaits dont les hommes vous ont comblé63 $»$.

\section{Dans un second, il déclare :}

«Une de mes peines, c'est qu'Auguste Perret-à mon entendement l'unique architecte de l'époque ne soit pas l'architecte de la Gloire de Dieu, Fresque. (...) Ma joie c'est qu'Auguste Perret ait répondu à mon appel, mais trop tard; ma sottise avait enjambé les bornes de l'idiositie et comment ${ }^{64}{ }^{\prime}$.

L'archevêque de Paris, son éminence le cardinal Dubois, investigateur de la messe au cinéma, soutient l'artiste. Pathé filme cette rencontre dans l'atelier au 86, rue NotreDame-des-Champs en 1928 et annonce : «Marcel-Lenoir, le plus grand peintre religieux de l'époque nous reçoit dans son atelier de Montparnasse ».

En 1928, Emmanuel de Thubert publie dans Les cahiers de la Douce France, l'ouvrage de Marcel-Lenoir, peintre et fresquiste, ses écrits. L'artiste note alors :

«La fresque veut un esprit vierge, des connaissances définies, le don inné de la composition, et une conscience dans l'accompli égale à celle d'un maître-ouvrier. (... ) La muraille est de tous les supports-celui qui est le plus propice à l'improvisation. (...) Tout portrait intimiste dû aux fresquistes est mural, en ce sens qu'il est toujours empreint de religieux. Le religieux est à la fresque ce que le dessin est à la peinture : de l'indispensable ${ }^{65} \%$.

De par ses fresques, Marcel-Lenoir, chevaucheur d'idéal, fait communier l'humain et le divin. En homme indépendant, guidé par sa religion esthétique, il lègue une œuvre 
personnelle, moderne, qui se distingue parmi l'ensemble du patrimoine de la peinture à fresque.

\section{NOTES}

1. - Je tiens à remercier Claire Vignes-Dumas, Marie Monfort, Laurence de Finance, ainsi que Madame le Professeur Luce Barlangue. Cet article prend appui sur des recherches menées en thèse de doctorat d'histoire de l'art contemporain à l'Université Toulouse II-Le-Mirail : MarcelLenoir et son temps.

2. - NAMY, Marie-Ange. Couronnement de la Vierge. Institut Catholique de Toulouse. Toulouse : Les éditions du Musée Marcel-Lenoir, 2008 (catalogue d'exposition). NAMY, Marie-Ange. MarcelLenoir, le cantique des mains, un peintre de Montparnasse. Église Notre-Dame-des-Champs à Paris. Toulouse : Les éditions du Musée Marcel-Lenoir, 2010 (catalogue d'exposition). NAMY, MarieAnge. « Le couronnement de la Vierge, Toulouse : le paradis mystique de Marcel-Lenoir ». L'Auta, 2011, n³0, p. 368-376.

3. - THUBERT de, Emmanuel. «Fresques modernes fresque éternelle». La construction moderne, $1935, n^{\circ} 3$, p. 53.

4. - COLLECTIF. Puvis de Chavannes au Musée des Beaux-Arts de Lyon. Lyon: Réunion des musées nationaux, 1998 (catalogue d'exposition).

5. - RIVET, Luce. « À propos de Marcel-Lenoir ». Bulletin du Musée Ingres, 1990, n61-62, p. 79.

6. - LAVALLE, Denis, THUILLIER, Jacques. «À propos de l'art mural en France ». Revue de l'art, 1995, volume 108, n¹08, p. 10.

7. - GHIL, René. Marcel-Lenoir, étude lue devant la Société "L'Art pour tous " en l'atelier de Marcel-Lenoir (83, rue de la Tombe-Issoire, Paris). Paris : Société « L’Art pour tous », 1906, p. 10.

8. - Archives Fondation Coubertin : manuscrit inédit: Les confessions de l'enlumineur Marcel-Lenoir, n.d.

9. - BOISSIER, Émile. L'enlumineur Marcel-Lenoir, l'homme et l'œuvre. Paris : Arnould, 1899, p. 98.

10. - Anonyme. «L'exposition des œuvres de Marcel-Lenoir ». La petite Gironde, 1903.

11. - RAMEY, Henri. «Après la manifestation de Bruniquel en l'honneur du peintre MarcelLenoir ». La Dépêche, 1949.

12. - SOULA, Camille. Dans GUILHEM, A. Marcel-Lenoir. Paris : Eugène Figuière Éditeur, 1919, p. 7.

13. - Archives BnF : Manuscrits Occidentaux, Fonds Stanislas Fumet, lettre de Marcel-Lenoir à Stanislas Fumet, Bruniquel, 1916.

14. - Archives BnF : Manuscrits Occidentaux, Fonds Stanislas Fumet, Correspondances MarcelLenoir, lettre de 1916.

15. - GIROU, Jean. « Marcel-Lenoir et la fresque de l'Institut Catholique de Toulouse ». Des lignes et des couleurs. Toulouse : Collège d'Occitanie, n.d., p. 87.

16. - POULAIN, Gaston. « Marcel-Lenoir ». A.B.C Magazine d'art, 1926, p. 84.

17. - COLLECTIF. Fernand Léger: la poésie de l'objet, 1928-1934. Centre Georges Pompidou, Musée national d'art moderne. Paris: RMN, 1981, p. 19 (catalogue d'exposition).

18. - ALLARD, Roger. "Manifestation d'atelier». Le nouveau spectateur, 1920, numéro 17-18, p. 128-129.

19. - Archives Musée Marcel-Lenoir : Manifestation d'atelier du 25 janvier au 20 février, 115 rue Notre-Dame-des-Champs, 1920. 
20. - Archives Musée Marcel-Lenoir : Manifestation d'atelier du 25 janvier au 20 février, 115 rue Notre-Dame-des-Champs, 1920.

21. - Archives Musée Marcel-Lenoir : Manifestation d'atelier du 25 janvier au 20 février, 115 rue Notre-Dame-des-Champs, 1920.

22. - Pierre Claude Dubois (1886-1972) né à Bar-le-Duc en Meuse, élève des Beaux-Arts et des Arts décoratifs, lié à l'Académie de la Grande Chaumière, à l'Académie Ranson, aux Ateliers d'art sacré, rencontre l'artiste à Montparnasse et devient son élève.

23. - Jean Charlot (1898-1979) est un peintre franco-mexicain qui apprend la technique de la fresque auprès de Marcel-Lenoir qu'il vénère, puis émigre au Mexique en 1921 en compagnie de son compatriote Diego Rivera.

24. - Licenoski-Lasar (1901-1964) est un artiste majeur du paysage yougoslave. En France, il fréquente l'École des Arts et Métiers du professeur Paul Baudouin, la Grande chaumière, l'atelier d'André Lhote. Ses études terminées à Belgrade, il arrive à Paris en 1927 afin de perfectionner la technique de la peinture murale et de la fresque. Lors de l'exposition de peintres yougoslaves à laquelle il participe composée de Milunovitch, Dobrovitch, le sculpteur Stiyovitch, Loubarda, Meguelouvitch à l'Institut des études slaves, rue Michelet, il rencontre Marcel-Lenoir.

25. - L'artiste Jozo Kljakovic (1889-1969) est une figure reconnue du paysage artistique croate, peintre, et également professeur de dessin, puis entre 1921 et 1943, enseigne la peinture murale à l'Académie des Beaux-Arts de Zagreb. Il étudie dans plusieurs écoles : à Zagreb, à Prague, à Vienne, à Rome, à Genève. Lors de ses séjours parisiens, il fréquente le milieu artistique, l'Académie Ranson où il retrouve Maurice Denis. Il remporte un prix lors de l'exposition internationale des Arts Décoratifs de Paris en 1925 pour ses fresques et ses vitraux.

26. - Le croate Ivo Rezek (1898-1979) est reconnu comme un artiste régionaliste. Après ses études en Croatie, puis à l'Académie de Prague, il séjourne à Paris dès les années 1924. Il participe au Salon d'Automne, et Salon des Tuileries.

27. - Antonio Gomide est une figure réputée du monde artistique brésilien. Il est né à Itapetininga dans l'État de Sao Paulo le 3 août 1895, et décédé le 31 août 1967 à Sao Paulo. Artiste pluridisciplinaire, il est à la fois peintre, sculpteur, décorateur et également scénographe. Il compte parmi ses amitiés des figures majeures de l'art moderne brésilien; ensemble, ils participent au renouveau de l'art de leur pays et incarnent l'âme brésilienne moderne. Après plusieurs voyages au Brésil, il se dirige vers l'Europe : entre la Suisse et la France, Lyon et Paris. Antonio Gomide s'installe à Paris, rue Saint-Jacques en 1921. VERNASCHI, Elvira. Gomide. Sao Paulo : Ediçoes de arte, 1989, p. 37-38, 77, 213.

28. - BURRUS, Christina. Diego Rivera, Frida Kahlo. Fondation Pierre Giannada. Martiny : Fondation Pierre Giannada, 1998, p. 20 (catalogue d'exposition).

29. - FAUCHEREAU, Serge. Les peintres révolutionnaires mexicains. Paris : Messidor, 1985, p. 56.

30. - DEFIANAS, Louis. « Un artiste yougoslave : Licenoski-Lasar ». Le miroir du centre, 1982, $n^{\circ} 164$, p. 11.

31. - CHIRICO de, Gorgio. Jozo Kljakovic. Rome : Éd. Univ., 1947.

32. - KLJAKOVIC, Jozo. U suvremenom Kaosu : uspomene i dozivljaji. Croatie : Matica hrvatska, 1992, p. 155-156.

33. - VERNASCHI, Elvira. Gomide. Sao Paulo : Ediçoes de arte, 1989, p. 37-38, 77, 213.

34. - Archives Musée Ingres : dossier Marcel-Lenoir. Maurice Albe est professeur de dessin d'art dans des établissements scolaires de la Dordogne. Il est professeur et directeur de l'École municipale de Dessin et Arts Décoratifs de Périgueux de 1947 à 1990. À partir de 1926, il expose au Salon des Indépendants ainsi qu'au Salon d'Automne à Paris. Il reçoit le diplôme d'argent de la Société Arts, Sciences, Lettres de Paris en 1972. Il est décoré de l'Ordre national du Mérite en 1973. BORDES, François. Maurice Albe, un artiste et son pays. Tour de Vésone à Périgueux : Éditions Fanlac, 1996, p. 26.

35. - HOSTEL (D'), Banville. « Les expositions de guerre ». Revue, 1917. 
36. - HOSTEL (D'), Banville. «Les expositions de guerre ». Revue, Paris, 1917, p. 20.

37. - CORNIN, Michel. Le théâtre de recherche entre les deux guerres: Le Laboratoire Art et Action. Paris : L'Age d'homme/La cité, 1974, p. 67.

38. - Celle-ci semble jouer d'un instrument peu visible sur la photographie. Marcel-Lenoir renouvelle la mythologie grecque : la déesse évoque peut-être à la fois Thalie muse de la comédie, Calliope celle de la poésie épique et de l'éloquence, Erato celle de la poésie lyrique et érotique, ou encore Euterpe celle de la musique, elle peut rappeler également Melpomène pour le chant et la tragédie, Polymnie pour la rhétorique. Ou bien Vénus pour la mythologie romaine.

39. - Archives Musée Marcel-Lenoir : photographie en noir et blanc, façade Autant Lara.

40. - AUTANT LARA, Claude. La rage dans le cœur, chronique cinématographique du $20^{\circ}$ ème siècle. Paris : Henri Veyrier, 1984, p. 39-40.

41. - SANCHEZ, Pierre. Dictionnaire du Salon d'Automne. Dijon : L'échelle de Jacob, 2006, p. 848.

42. - Archives privées : lettre de Marcel-Lenoir adressée à Paul Rolland, 1920.

43. - Archives privées : lettre de Marcel-Lenoir adressée à Paul Rolland, 1920.

44. - COLLECTIF. Lignes. Lyon : Les deux collines, Lyon, 1920.

45. - PORTAL, Frédéric. Des couleurs symboliques dans l'Antiquité, le Moyen-Age et les temps modernes. Paris : Treuttel et Wurtz, 1837.

46. - BRICON, Étienne. « Le salon d'Automne ». Gazette des Beaux-Arts, 1920.

47. - PLEE, Léon. "Le salon d'Automne». Les annales politiques et littéraires, revue universelle, illustrée, hebdomadaire, 1920, n¹948.

48. - GYBAL, André. « Le salon d'Automne, Paris 1920 ». L'art libre, 1920.

49. - Archives municipales de Montricoux : lettre de Marcel-Lenoir au père Pierre Augé, de Paris, datée du 16 décembre 1922.

50. - Archives municipales de Montricoux : lettre du maire Lasserre à Marcel-Lenoir du $1^{\mathrm{er}}$ mars 1923.

51. - Archives municipales de Montricoux : souscription du 23 février 1923.

52. - Archives municipales de Montricoux: lettre du père Pierre Augé au maire Monsieur Lasserre et aux conseillers municipaux, datée du 10 janvier 1923.

53. - SANCHEZ, Pierre. Dictionnaire du Salon d'Automne. Dijon : L'échelle de Jacob, 2006, p. 848.

54. - VANDERPYL, Fritz-René. «Le vernissage de la société nationale ». Le petit parisien, 1921, n 16114.

55. - DENOINVILLE, Georges. "Salon de la société nationale des Beaux-Arts-Considérations générales, les portraits et les figures ». La revue des Beaux-Arts, 1921, n³55.

56. - JULLIAN, René, BERNARD, Jean, STONESCO, Lucien, GREMONT-GERVAISE, Pascale. Joseph Bernard. Saint-Rémy-lès-Chevreuse : Fondation de Coubertin, 1989.

57. - TESTARD, Maurice. L'homme à l'hortensia. Paris : Vigot, 1955, p. 55.

58. - SALMON, André. Montparnasse, mémoires. Paris : Arcadia, 2003, p. 61.

59. - SOULA, Camille. "Le Couronnement de la Vierge de Marcel-Lenoir ». La Renaissance des arts français et des industries de luxe, $1923, \mathrm{n}^{\circ} 8$.

60. - RIVET, Luce. «À propos de Marcel-Lenoir... ». Bulletin du Musée Ingres, 1989, n61-62, p. 76.

61. - SOULA, Camille. "Le Couronnement de la Vierge de Marcel-Lenoir». La Renaissance des arts français et des industries de luxe, 1923, n8. FUMET, Stanislas. « Marcel-Lenoir et la fresque ». L'art et les artistes, 1924. VAUDOYER, Jean-Louis. "Marcel-Lenoir». Art et décoration, 1924. BRILLANT, Maurice. « L'art-L'art religieux en 1922-1923 ». L'Almanach Catholique français, 1924, p. 263-270.

62. - POULAIN, Gaston. « Un grand peintre méconnu-Marcel-Lenoir est mort ». Comoedia, 1931, p. 1-2.

63. - Archives Musée Marcel-Lenoir: Manifestation d'atelier du $1^{\mathrm{er}}$ au 31 mars, avec annonce intitulée par l'artiste : « À propos de l'ordonnance « À la Gloire de Dieu » (fresque) », vers 1928.

64. - Archives Musée Marcel-Lenoir : Manifestation d'atelier du $1^{\mathrm{er}}$ au 31 mars, vers 1928. 
65. - MARCEL-LENOIR. Marcel-Lenoir, peintre et fresquiste, ses écrits. Paris : Cahiers de la Douce France, 1928, p. 36, 62, 101.

\section{RÉSUMÉS}

Dans le paysage artistique de la fin du XIX et de la première moitié du XX ${ }^{e}$ siècle, Marcel-Lenoir (1872-1931) compte dans la réécriture de l'histoire de l'art. En effet, cet artiste prolifique, bijoutier à ses débuts, puis enlumineur, graveur, peintre et fresquiste, bénéficie à l'époque d'une aura certaine, auprès de critiques, d'historiens, de littérateurs, d'artistes et de photographes. Puis il fait l'objet d'un oubli, et enfin d'une renaissance progressive. Il s'agit de se concentrer ici sur un visage particulier de Marcel-Lenoir : celui du fresquiste. Marcel-Lenoir, du bijou à la fresque, inscrit son nom au cœur du renouveau de l'art sacré et de la résurrection de la fresque. Entre 1919 et 1920, il crée l'Institut d'esthétique contemporaine en son atelier, 86 rue NotreDame-des-Champs, destinée à l'enseignement de la fresque, où il reçoit alors de nombreux élèves français et étrangers. Entouré de quelques-uns d'entre eux, il réalise entre 1920 et 1923, pour l'Institut Catholique de Toulouse, une fresque monumentale de $63 \mathrm{~m}^{2}$ célébrée dans le monde des arts : Le Couronnement de la Vierge. Celle-ci est inscrite à l'Inventaire supplémentaire des Monuments historiques depuis le 13 février 1996. En outre, le fresquiste connaît à l'époque une certaine notoriété grâce au succès suscité par L'Annonciation, conservée en l'église de Montricoux. Enfin, il s'exerce à d'autres projets de fresques, de grandes et petites dimensions, peu connues, détruites, inachevées, tombées dans l'oubli, tel la Femme au ruban rouge des réserves du Musée National d'Art Moderne, Centre Georges-Pompidou. Dans ses fresques, Marcel-Lenoir, chevaucheur d'idéal, fait communier l'humain et le divin. En homme indépendant, guidé par sa religion esthétique, il lègue alors une œuvre personnelle, moderne, qui se distingue parmi l'ensemble du patrimoine dédié à la fresque.

In the artistic landscape of the late nineteenth and the first half of the twentieth century, Marcel-Lenoir (1872-1931) included in the rewriting of the history of art. Indeed, this prolific artist, jeweler in its infancy then miniaturist, engraver, painter and muralist, has to be a certain age, with critics, historians, writers, artists, and photographers. Then there has been an oversight, and finally a gradual renaissance. Here is a focus on a particular face of Marcel-Lenoir: that of fresco. Marcel-Lenoir, from jewel to fresco, his name is at the heart of the revival of sacred art and the resurrection of the fresco. In 1920, he founded the Institute of contemporary aesthetics in his workshop, 115, rue Notre-Dame-des-Champs, for teaching the fresco, where he receives many French and foreign students. Surrounded by some of them, he made between 1920 and 1923 for the Catholic Institute of Toulouse a monumental fresco of 90 square meters celebrated in the world of art: The Coronation of the Virgin. This is included in the supplementary inventory of historical monuments since February 13, 1996. In addition, the fresco knew at the time a certain notoriety thanks to the success generated by the Annunciation, preserved in the church of Montricoux. Finally, he worked on other projects of frescoes, large and small, little known, destroyed, incomplete, fallen into oblivion, as the Red Ribbon Woman stored in the reserves of the Musée National d'Art Moderne, Centre Georges Pompidou. In his paintings, Marcel-Lenoir, Rider of ideal communion is human and divine. As an independent man, guided by his religion aesthetics, he left then a personal work, modern, distinguished among all the assets dedicated to the fresco. 
INDEX

Mots-clés : art contemporain, $19^{\circ}$ et $20^{\circ}$ siècle, art sacré, fresque, compositions murales monumentales et décoratives, art monumental, art décoratif monumental, peinture, symbolisme, cubisme, futurisme, manifeste, Marcel-Lenoir

\section{AUTEUR}

\section{MARIE-ANGE NAMY}

Doctorante en Histoire de l'art contemporain à l'Université Toulouse-II-Le-Mirail, membre de FRAMESPA/UMR 5136, chargée de conservation du Musée Marcel-Lenoir marie-ange@marcellenoir.com 